\title{
UNA APROXIMACIÓN AL ESTUDIO DE LOS CONSUMOS FINANCIARIZADOS DE LOS SECTORES POPULARES DE BUENOS AIRES, ARGENTINA ${ }^{1}$
}

\author{
AN APPROACH TO THE STUDY OF FINANCIAL CONSUMPTION IN THE LOW-INCOME \\ SECTORS OF BUENOS AIRES, ARGENTINA
}

\section{Renato Mauricio Fumero² \\ lara Nadina Hadad ${ }^{3}$}

\begin{abstract}
Resumen
En el presente artículo proponemos un abordaje sobre los consumos financiarizados de los sectores populares en Argentina. Como han destacado múltiples trabajos realizados en diferentes países latinoamericanos, el estudio de este fenómeno, reciente y muy extendido, exige poner en suspenso algunos de los lugares comunes que han fundamentado la caracterización de la vida económica de los sectores populares. Proponemos aquí algunas direcciones teóricas para repensar estos consumos y presentamos el análisis de los resultados de una investigación, aún en curso, sobre consumo y endeudamiento en sectores populares del área metropolitana de Buenos Aires.
\end{abstract}

Palabras claves: consumo; finanzas; sectores populares; economía popular

Doi: http://dx.doi.org/10.15359/eys.22-52.3

Fecha de recepción: 16-02-2017. Fechas de reenvíos: 24-03-2017, 29-05-2017, 14-07-2017, 18-07-2017, 21-082017, 01-09-2017. Aceptado el 05-09-2017. Publicado el 11-09-2017.

${ }^{1}$ Investigación realizada con el auspicio del Centro de Investigaciones Socio-Económicas de Buenos Aires (CISBA), actualmente denominado Centro de Estudios Económicos y Sociales, perteneciente al Banco de la Provincia de Buenos Aires. Proyecto: Hacia una economía de los sectores populares: Desafíos conceptuales y políticos. El trabajo de campo se realizó entre los años 2013 y 2014 en distintos partidos de la provincia de Buenos Aires, Argentina.

2 Sociólogo, Investigador, Consejo Nacional de Investigaciones Científicas y Técnicas (CONICET)/ Instituto de Altos Estudios Sociales (IDAES), Universidad Nacional de San Martín (UNSAM), Buenos Aires, Argentina. Correo electrónico: rmfenidaes@gmail.com

3 Socióloga, Investigadora, Instituto de Altos Estudios Sociales (IDAES), Universidad Nacional de San Martín (UNSAM), Buenos Aires Argentina. Correo electrónico: hadadiara@gmail.com 48

Renato Mauricio Fumero e lara Nadina Hadad

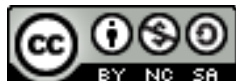

Revista Economía y Sociedad by Universidad Nacional is licensed under a CreativeCommons Reconocimiento-NoComercial- 


\begin{abstract}
An approach is proposed on the study of financial consumption in the low-income sectors of Argentina. As mentioned in multiple research studies conducted in different Latin American countries, the study of this recent and widespread phenomenon demands to highlight some of the common places that have caused the characterization of the economic life of the popular sectors. We propose some theoretical guidelines to reconsider such consumption and analyze the results of our still ongoing investigation regarding consumption and indebtedness in the lower sectors of the metropolitan area of Buenos Aires.
\end{abstract}

Keywords: consumption; finance; popular sectors; popular economy

\title{
Introducción
}

Si bien el concepto de "financiarización" se presenta amplio e impreciso, su eventual falta de rigurosidad permite englobar abordajes y análisis que privilegian diversos aspectos, dimensiones, escalas y metodologías. A propósito de esto, Van der Zwan (2014) ha elaborado un estado del arte sobre la noción de financiarización (financialization) en la que destaca tres enfoques. Uno de ellos consiste en entender la financiarización como régimen de acumulación; el otro refiere a los cambios en las organizaciones y en la gestión corporativa moderna y, por último, un tercer acercamiento se centra en las experiencias de las finanzas al nivel de las prácticas de los sujetos. En líneas generales, podríamos decir que interrogar el concepto de financiarización apunta a elaborar nuevas categorías y herramientas conceptuales que ayuden a entender las transformaciones del capitalismo contemporáneo. En particular, Epstein (2005) propone entender por financiarización las transformaciones del capitalismo mundial en términos de globalización financiera y, con ello, la expansión e intromisión de las finanzas en todos los ámbitos de la vida social.

En este trabajo nos proponemos analizar las modalidades específicas que adquiere la financiarización de los consumos de los sectores populares en la Argentina actual. Los procesos de creciente financiarización a través de los cuales se genera una parte significativa de las prácticas económicas cotidianas de las personas constituyen una dimensión compleja y central a la hora de comprender las actuales formas de consumo de los distintos sectores sociales; entre ellos, las clases populares. Tanto a nivel local como regional, diversos estudios han señalado la importancia de analizar las redes monetarias que tejen las economías y los presupuestos domésticos de los grupos sociales de menores recursos (Roig, 2015; Figueiro, 2013; Wilkis, 2013, 2014; Hornes, 2014; Müller, 2009; Villarreal, 2004; Ossandón, 2011; Ossandón, Ariztía, Barros y Peralta, 2012; entre otros). Estos trabajos han buscado dar cuenta del grado de monetización en el que se inscriben las prácticas y relaciones sociales del universo popular, muchas veces 
invisibilizado por abordajes centrados en la economía de la pobreza, la subsistencia, la exclusión y la informalidad.

Nuestra reflexión se inscribe en los últimos años, correspondientes al amplio período de gobiernos kirchneristas (2003-2015), en los que el consumo interno y masivo fue considerado pilar del crecimiento económico. Los datos del Banco Central de la República Argentina (BCRA) señalan que durante estos años el grueso del stock total de crédito otorgado a empleados en relación de dependencia se ha compuesto de préstamos personales y con tarjeta de crédito, quedando en segundo lugar los prendarios y representando los créditos hipotecarios apenas un 10\% (Bercovich, 2016) ${ }^{4}$. Esta recomposición del mercado de crédito implicó una diversificación y segmentación de la oferta crediticia que de la mano de determinadas políticas públicas propulsó el consumo y endeudamiento de los sectores de menores ingresos, tradicionalmente no bancarizados pero sí alcanzados por estructuras financieras no reguladas por la autoridad monetaria ${ }^{5}$. Marcando un paralelismo con lo que sucedía en los sectores medios, para el año 2009, una encuesta del BCRA constataba que dentro del "top five" de productos ofrecidos a los sectores bajos por las entidades financieras no reguladas ${ }^{6}$ se encontraban los siguientes: préstamos personales (57\%), préstamos de bajo monto (54\%), tarjeta de crédito (28\%), microcréditos (17\%), caja de ahorro (10\%).

En el marco de esta coyuntura, cabe preguntarse: ¿en qué condiciones se realiza ese consumo?, ¿a través de qué medios?, ¿a qué costos? En términos analíticos, el consumo deviene una puerta de entrada para reconstruir las especificidades de las economías populares en sus vinculaciones con las características que presentan los sectores más dinámicos de la economía capitalista. La financiarización -entendida como el uso de tarjetas de compra, tarjetas de crédito y créditos personales- es un indicador del modo en que lo financiero se conecta con lo popular ${ }^{7}$.

\footnotetext{
${ }^{4}$ Tomando a la familia asalariada promedio como parámetro, durante los gobiernos de Néstor y Cristina Kirchner el endeudamiento osciló entre un sueldo y medio (2003 y 2004) y dos sueldos (2008, 2014 y 2015), con picos máximos de 2,2 sueldos (2007 y 2013).

${ }^{5}$ A la expansión de la oferta crediticia en manos de entidades financieras reguladas y no reguladas por el BCRA a partir del año 2003 hay que agregar la batería de políticas públicas impulsadas desde el Estado (reconversión de planes sociales en contraprestaciones monetarias, $\mathrm{AUH}$, etc.) que tendieron a incentivar el consumo interno en un escenario de reconfiguración del mundo del trabajo; escenario caracterizado por la consolidación de formas autogestivas y cooperativas de anclaje territorial y producción a pequeña escala.

${ }^{6}$ La muestra de entidades financieras no reguladas construida en la investigación se componía de 25 cooperativas de crédito, 55 asociaciones mutuales, 3 financieras, 11 entidades de microfinanzas y 18 tarjetas de crédito no bancarias.

${ }^{7}$ La reconfiguración del crédito hacia la banca personal es un fenómeno que no solo se manifiesta en el sistema financiero argentino, sino a nivel mundial, y que va acompañado de una gran rentabilidad en la medida en que -en comparación con otras categorías-, se trata de préstamos de corto plazo, menores montos y mayor interés; todo lo cual redunda en un menor riesgo para los inversores y las entidades oferentes. Por otra parte, cabe destacar que el crédito a las familias se canaliza a través de oferentes de diversa categoría. Así, es posible distinguir entre (i) entidades enmarcadas en la Ley de Entidades Financieras (bancos, cajas de crédito y otras compañías financieras), (ii) compañías de crédito al consumo no enmarcadas en dicha ley y, por lo tanto, no reguladas por el Banco Central 50
}

Renato Mauricio Fumero e lara Nadina Hadad

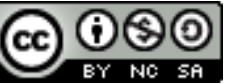

Revista Economía y Sociedad by Universidad Nacional is licensed under a CreativeCommons Reconocimiento-NoComercial- 
A la vez, no es posible comprender o dimensionar las implicancias sociales y políticas de la ampliación del consumo sin posar la mirada sobre las particularidades que asumen las economías populares e informales. Investigaciones regionales como la de Cielo y Antequera (2012), en Bolivia, o las de Giraldo (2013), en Colombia, advierten sobre las múltiples relaciones no lineales que vinculan la materialidad de los espacios de la economía popular con la totalidad social y la economía general.

En efecto, la economía popular como fenómeno social engloba un sinfín de prácticas y lógicas que no se desarrollan por fuera del "mercado" o en contra del capitalismo, sino en sus pliegues e intersticios (Figueiro, 2016). Numerosas investigaciones han evidenciado las múltiples conexiones entre la formalidad e informalidad, la legalidad e ilegalidad, lo local e internacional, lo mercantil y lo personal, que atraviesan las formas laborales, las economías domésticas y las prácticas de consumo y gasto que se configuran en el universo popular (Wilkis y Roig, 2015; Müller, 2014; Gago, 2012, 2013; entre otros).

En la primera sección de este trabajo presentamos algunas de las ideas principales que orientan teóricamente nuestra investigación. Sostenemos que el análisis de las prácticas de consumo financiarizado ofrece un vía de acceso fundamental para pensar la economía popular desde un punto de vista renovado. En la segunda sección presentamos el análisis de los resultados parciales de la investigación empírica ${ }^{8}$ de la que estamos participando. La primera que, con un enfoque cuantitativo, interpela los consumos financiarizados de los sectores populares en la Argentina, desde una perspectiva atenta a los desarrollos recientes de la sociología económica en la región. Acompañamos el análisis de estos datos con algunas hipótesis que pretenden proyectar una posible agenda de investigación futura.

\section{Sección primera: algunas ideas en torno a los consumos financiarizados de los sectores populares}

\section{Sobre la economía popular}

La definición de la economía popular dependió durante mucho tiempo, fundamentalmente, del modo en que fue pensada su integración (funcional o no funcional) al régimen de acumulación capitalista (Chena, 2017). La reflexión en torno a la pobreza, primero, y en torno a la exclusión

ni por otro organismo de control interviniente en el sistema financiero, (iii) cooperativas y asociaciones mutuales, y (iv) tiendas comerciales minoristas. Un rasgo distintivo del segmento de crédito al consumo es que varios de los principales bancos argentinos de capital privado mantienen el control accionario de compañías especializadas en consumo (empresas emisoras de tarjetas de crédito, compañías financieras, compañías de microfinanzas, entre otros). (Balzarotti y Anastasi, 2013).

${ }^{8}$ Los datos producidos surgen del proyecto "Hacia una economía de los sectores populares: Desafíos conceptuales y políticos", llevado adelante por el equipo de trabajo del Centro de Estudios Económicos y Sociales, perteneciente al Banco de la Provincia de Buenos Aires. 
social (Kessler, 2014), luego, sirvió como criterio de demarcación para definir las prácticas y los actores que formaban parte de aquella región de la economía que se caracterizó como "informal".

El desarrollo de la categoría "informalidad" ha permitido, en diferentes momentos históricos, caracterizar a la economía popular, por oposición a la "economía formal”, a partir de fenómenos tales como los bajos ingresos, la precariedad laboral, la escasa productividad y la falta de innovación. Este tipo de perspectiva condujo mayormente a postergar el análisis detallado de algunas de las prácticas e instituciones económicas de los sectores populares. El estudio de los consumos y de la vida financiera de estos sectores, por caso, ha merecido mucha menor atención que el estudio de sus ingresos y su actividad laboral.

La perspectiva teórica que fundamenta nuestra indagación sobre los consumos financiarizados de los sectores populares fija un punto de partida alternativo para pensar la economía popular, que no se apoya en la oposición dicotómica entre economía formal e informal. Proponemos pensar la economía popular en la intersección de dos órdenes de interrogación complementarios. En este sentido, la economía popular se considera:

I) Como una posición objetivada dentro de la estructura social a partir del sistema de precios (Roig, 2017).

II) Como una serie de prácticas sociales que producen formas específicas, institucionales y subjetivas de participar en la trama social de intercambios económicos.

La apuesta teórica que se plantea desde esta línea de trabajo es descubrir a través del estudio de las prácticas e instituciones específicas, las tramas fiscales, financieras, laborales y de consumo que condicionan el posicionamiento estructural (Ibíd.) y la construcción subjetiva de los colectivos estudiados al interior de sociedades marcadamente desiguales. Los ingresos percibidos y la inclusión fiscal ${ }^{9}$ no deben ser analizados al margen de los costos financieros, las modalidades de consumo y los precios relativos que afrontan los sectores populares.

\section{Sobre los consumos financiarizados como clave interpretativa}

En particular, sostenemos que el estudio de los consumos y de la vida financiera contribuye fundamentalmente a la comprensión actual de los sectores populares. El consumo puede transformarse en una puerta de entrada para reconstruir las especificidades de la economía popular, estudiar las relaciones que mantiene con los otros sectores de la economía y descubrir la fisonomía local del capitalismo contemporáneo. Al mismo tiempo, los consumos permiten

\footnotetext{
${ }^{9}$ Giraldo (2017) coincide en remarcar que los trabajadores de la economía popular se encuentran por fuera del contrato social que se expresa por medio de la fiscalidad; es decir, de aquellas garantías y prestaciones sociales ofrecidas por el Estado a cambio del pago de impuestos o tributos.
}

52

Renato Mauricio Fumero e lara Nadina Hadad

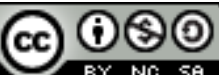

Revista Economía y Sociedad by Universidad Nacional is licensed under a CreativeCommons Reconocimiento-NoComercial- 
vislumbrar la trama financiera de la que participan los sectores populares. Consideramos que es necesario preguntarse, entonces, en qué condiciones (qué, dónde y cuándo), a través de qué medios y a qué costos consumen estos sectores.

Si bien la preocupación por el consumo ha estado muy presente en los debates sobre la situación económica argentina reciente, en términos de problemática social muchas veces quedó subordinado a la interrogación sobre el "poder de compra" del ingreso, que aparece como la dimensión más confiable para evaluar el bienestar social. Desde este punto de vista, la atención sobre los sectores populares queda fuertemente determinada a partir de la presunción de que ellos comprometen, en mayor medida y de modo más urgente, sus consumos a la satisfacción de sus necesidades básicas. Es decir, un agente que, en principio, no gestiona excedentes ni participa de circuitos financieros que no estén ligados a la subsistencia. Al fin y al cabo, ¿quién le prestaría dinero a quien no tiene capacidad de ahorro? y ¿cómo sería capaz de prestar dinero este a otros si todo su dinero está comprometido en las consumos básicos?

Estos presupuestos, formulados originalmente como principios explicativos, tienden habitualmente a convertirse, a partir de una serie de deslizamientos, en los puntos de apoyo valorativos de una consideración moral sobre los usos que los sectores populares hacen del dinero. Específicamente, fundamentan juicios normativos que cuestionan las decisiones de consumo que estos adoptan. Los gastos que los sectores populares orientan al consumo de bienes no básicos son juzgados como "irracionales" (Figueiro, 2013) o moralmente "reprobables" (Wilkis, 2013).

En términos teóricos, no advertir la necesidad de pensar la gestión y destrucción de excedentes (Bataille, 2008) como una dimensión central -y lógicamente autónoma- para pensar el consumo, limita la posibilidad de comprender la vida económica de los sectores populares. El consumo es una práctica social compleja que tiene una dinámica autónoma. De hecho, el consumo avanza sobre las fronteras de los ingresos y se proyecta en formas de endeudamiento que responden a racionalidades refractarias a la "formalización económica" (Polanyi, 1976). Surge, entonces, la necesidad de dislocar los marcos preconcebidos que pretenden regir teórica y moralmente las articulaciones entre consumo, ingreso, ahorro y deuda, y avanzar en enlaces empíricamente fundamentados entre estas nociones; algo no solo demandado para el caso de los sectores populares, claramente.

La financiarización de los consumos populares puede ser rastreada en una serie de prácticas que muestran como "lo popular" participa de las tramas financieras: uso de tarjetas de compra, tarjetas de crédito y créditos personales, además de acceso a préstamos de dinero y de tarjetas entre familiares y amigos. Ahondar en ello resulta necesario para comprender el sentido que hoy tiene hablar de "economía popular", debido, al menos, a dos motivos: por un lado, porque los vínculos financieros muestran formas particulares en que se configura la existencia económica de los sectores populares; por otro lado, porque estos vínculos financieros también 
expresan rasgos del funcionamiento sistémico de las finanzas en el conjunto de la economía. Estudiar el modo en que se realizan los consumos en la economía popular permite, en suma, dar cuenta de las formas de dominación que trama el sector financiero y de las estrategias sociales frente a estas (De Certeau, 1996), así como también, finalmente, permite caracterizar la posición estructural que ocupan los sectores populares. Las condiciones asimétricas, a través de las cuales los sectores populares acceden a las mediaciones financieras y a los intercambios comerciales, convergen para determinar estructuralmente la singularidad de la economía popular frente a otros sectores de la sociedad (por ejemplo, las clases medias).

\section{Sección segunda: la investigación y sus resultados}

\section{Análisis de los datos preliminares}

Hace apenas 25 años, cuando Deleuze (1995) proclamaba al "hombre endeudado" como figura subjetiva de las sociedades contemporáneas, aun pensaba que tres cuartas partes de la humanidad seguirían siendo "...demasiado pobres para endeudarlas..." (p. 283). Recientemente, siguiendo la misma estela de pensamiento, Lazzarato (2013) consideró la relación deudoracreedor como la forma contemporánea de la lucha de clases. Podríamos imaginar que entre estas dos referencias media un salto epocal: el desarrollo del capitalismo financiero como régimen global de acumulación.

Como exponen diversos trabajos ${ }^{10}$, este proceso se ha manifestado como un ciclo de transformaciones a escala latinoamericana. El proceso de masificación del consumo a crédito ha implicado tanto la extensión y fortalecimiento de prácticas y saberes institucionales preexistentes como el desarrollo de instituciones nuevas. Por instituciones no nos referimos exclusivamente a las instancias legalmente reconocidas como tales (tarjetas de crédito, créditos al consumo, etc.), sino también a aquellas no formalizadas que operan simultáneamente -de manera integrada o paralela- al sistema legalmente sancionado (por ejemplo, los préstamos monetarios entre familiares y amigos, las estrategias familiares para acceder al sistema de crédito, etc.). Es en relación con esta estructura de financiamiento que las prácticas concretas de consumo financiarizado de los sectores populares en la región adquieren su significado específico.

Los datos que presentamos a continuación surgen de la encuesta desarrollada dentro del proyecto "Hacia una economía de los sectores populares: Desafíos conceptuales y políticos" por el Centro de Investigaciones Socio-económicas de Buenos Aires (CISBA), y que hemos aplicado entre noviembre de 2013 y finales de 2014 en diferentes partidos de la provincia de Buenos

\footnotetext{
10 Sólo a modo de ejemplo y sin ánimo de exhaustividad, podemos mencionar a Ossadón (2011), Ossandón et al. (2012), Barros (2009), para el caso chileno; Kumar (2004) y Müller (2009), para el caso brasileño; Villarreal (2004), para el caso mexicano; Wilkis (2014), D'Onnofrio (2008) y Figueiro (2010), para el argentino; entre tantos otros. 54
}

Renato Mauricio Fumero e lara Nadina Hadad 
Aires ${ }^{11}$. Este proyecto participa de una agenda teórica común con los estudios regionales anteriormente mencionados. En particular, comparte con trabajos etnográficos, como los de Wilkis $(\underline{2013}, \underline{2014})$ y Figueiro $(\underline{2010}, \underline{2013})$, una serie de interrogantes y diagnósticos sobre la vida económica de los sectores populares pero, a diferencia de ellos, avanza en la construcción de una ambiciosa investigación cuantitativa.

La encuesta buscó relevar los consumos financiarizados de los sujetos interrogados, quienes, por sus características sociodemográficas, permiten inducir resultados más generales sobre la economía popular. Se buscó conocer el tipo de artículo, la forma de pago y las prácticas e instituciones financieras involucradas en estas transacciones. También se indagó sobre el nivel y tipo ${ }^{12}$ de ingresos que percibe esta población para poder ofrecer una caracterización más precisa de ella y del impacto que sobre su economía tiene el acceso a créditos o préstamos para el consumo.

Dividimos en dos secciones el análisis de los datos producidos. En la primera presentamos los datos referidos a los bienes y a la modalidad de pago; en la segunda avanzamos en la caracterización de las formas de financiación específicas.

\section{Datos referidos a los bienes y a la modalidad de pago}

Los datos producidos corresponden a 282 consumos de bienes y servicios no básicos. En términos sociodemográficos, la población encuestada se compone -en mayor medida- de trabajadores cooperativistas que integran el programa Argentina Trabaja ${ }^{13}$.

Como muestra la Tabla $\underline{1}$, las compras de electrodomésticos, ropa, celulares y muebles representan el $77 \%$ de los consumos recogidos. Solo los electrodomésticos representan el $41 \%$, y la ropa el $18 \%$ del total. Tomando este grupo de bienes es interesante observar que no todos ellos son adquiridos en la misma medida recurriendo a planes de cuotas. Así, se observa que mientras que el 76,5\% de las compras de electrodomésticos se hicieron en cuotas, en el caso de la ropa estas se emplearon en el $54 \%$ de los casos; y en cuanto a los celulares y los muebles, estos alcanzaron, respectivamente, al $57 \%$ y al $68 \%$ de los casos.

\footnotetext{
${ }^{11}$ Bella Vista, Berisso, Florencio Varela, General Rodríguez, La Plata, Lanús, Lomas de Zamora, Malvinas Argentinas, Máximo Paz, Moreno, Quilmes y Tigre son algunos de los lugares que han sido visitados.

${ }^{12}$ Aunque se trabajó con una población que es cooperativista y tiene un ingreso homogéneo, los trabajos adicionales comúnmente llamados "changas" y los programas sociales como la Asignación Universal por Hijo (AUH) redundan ingresos totales diferentes.

${ }^{13}$ El programa Argentina Trabaja, desarrollado por el Estado Nacional en relación con los gobiernos municipales, contaba, al momento de realizar la encuesta, con aproximadamente 150.000 inscritos en la provincia de Buenos Aires. El programa tiene como destinatarios a personas de hogares en situación de alta vulnerabilidad social y que no cuentan con otro ingreso proveniente de algún trabajo registrado, pensión u otro plan social. La mayoría de los beneficiarios (58,7\%) estaba en un rango de edad que iba desde los 25 a los 49 años, mientras que el $25,5 \%$ tenía entre 18 y 24 años. Solo el 19\% de los titulares había completado la educación formal.
} 
Tabla 1.

Adquisición de bienes y servicios no básicos y, relativos a cada uno, pago en cuotas

\begin{tabular}{lcc}
\multicolumn{1}{c}{ Producto } & Porcentaje - Consumos & Porcentaje - Pagado en cuotas \\
\hline Electrodomésticos & $41 \%$ & $77 \%$ \\
Ropa & $18 \%$ & $54 \%$ \\
Celular & $10 \%$ & $57 \%$ \\
Muebles & $8 \%$ & $68 \%$ \\
Otro & $7 \%$ & $71 \%$ \\
Materiales para & $4 \%$ & $42 \%$ \\
construcción & $3 \%$ & $63 \%$ \\
Computadora & $3 \%$ & $13 \%$ \\
Fiestas (15 y matrimonio) & $3 \%$ & $50 \%$ \\
Moto & $1 \%$ & $0 \%$ \\
Entierros & $100 \%$ & \\
Total general & &
\end{tabular}

Fuente: elaboración propia con base en la encuesta aplicada, Cisba. 2013-2014.

En términos de evaluar la extensión de los consumos financiarizados, estos datos y los restantes exhibidos muestran una participación elevada de la población analizada en estructuras de financiamiento. Con excepción de las categorías "Fiestas (15 y matrimonio)" y "Materiales de construcción", donde los valores son inferiores, y de "Entierros", donde no se registraron pagos en cuotas, para el resto de las categorías los consumos realizados en cuotas son en todos los casos superiores a la mitad del total relativo. Estos datos permiten afirmar una proposición que podría resultar contraintuitiva. Muestran que en dos de los ámbitos que requieren mayores erogaciones -como son las fiestas y los entierros- los pagos en cuotas son ostensiblemente menos frecuentes ( $13 \%$ y $0 \%$, respectivamente) que en consumos menos onerosos (por ejemplo, ropa). Las cuotas no aparecen como el destino necesario de los consumos más costosos, sino como un sistema del que participa cierto tipo de comercios que no necesariamente es el mismo en todos los sectores sociales. Del total de los consumos relevados, el $71 \%$ ha sido realizado a crédito, mientras que el $28 \%$ al contado. Distribución que, como vemos, no depende necesariamente del valor de los bienes en cuestión.

La canasta de consumos de los sectores populares exhibe una heterogeneidad de costos radical cuando se la contrasta con la de otros sectores sociales. Los bienes comercializados en La Salada (o en otras saladitas) (Gago, 2012) o en productos alimenticios circulan dentro de la economía popular a precios más accesibles (Roig, 2017). Los nuevos consumos de electrodomésticos, sin embargo, en relación con los presupuestos mensuales y a la inestabilidad laboral, presentan un diferencial de costos altísimo (Ibíd.). En relación con este punto, nuestra intención es mostrar que la presencia desigual de las formas de financiamiento en las diferentes áreas de consumo de la economía popular no responde inmediatamente a los precios de los bienes en cuestión. Tomando este dato en forma aislada, sería posible afirmar que, para el caso de los sectores 56

Renato Mauricio Fumero e lara Nadina Hadad

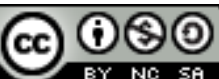

Revista Economía y Sociedad by Universidad Nacional is licensed under a CreativeCommons Reconocimiento-NoComercial- 
populares, los servicios (funerarios, fiestas, etc.) parecieran aún menos integrados a redes de financiamiento respecto de la oferta de bienes, especialmente tecnológicos.

Resulta necesario avanzar en el estudio del funcionamiento de las diferentes áreas de consumo de los sectores populares para entenderlas en su especificidad. Observamos, por ejemplo, que en momentos de recorte de los ingresos ${ }^{14}$ algunos encuestados comenzaban a endeudarse para comprar alimentos, lo que invita a incorporar fluctuaciones temporales. Observamos, asimismo, que algunos de los bienes adquiridos no se ofrecerían para el pago al contado, sino solo en cuotas, lo que exige relativizar la consideración sobre las formas de pago ${ }^{15}$.

\section{Caracterización de las formas de financiación específicas}

Los datos recogidos hasta el momento sobre consumo nos permiten evaluar la incidencia y las modalidades que adopta la financiarización para el caso de los consumos de los sectores populares. Por ejemplo, de la extensión de las redes de asistencia financiera entre familiares, amigos y vecinos nos habla el hecho de que casi un tercio de los encuestados (31\%) dijo prestar regularmente dinero a amigos o familiares. Este dato, que podría merecer diferentes ponderaciones, se torna particularmente elocuente cuando se lo contrasta con el modo en que el pensamiento económico obtura la posibilidad de gestión de excedentes para estos sectores. Entre quienes prestan dinero, el 51\% dice prestar hasta $\$ 200$, el 32\% presta entre $\$ 201$ y $\$ 400$ y el $17 \%$ restante presta más de $\$ 400$. La presencia de excedentes dentro del grupo analizado nos invita a especular a propósito de categorías y lógicas que instan a desacoplar aquello que el pensamiento económico ha enlazado, y a pensar que la lógica que gobierna el ahorro y la lógica que gobierna el consumo no son equivalentes ni complementarias, en la medida en que no están determinadas de igual forma por el ingreso.

Por otra parte, es posible perfilar algunas características estandarizadas de las formas de financiamiento a la que tiene acceso la población encuestada en sus consumos:

En el $97 \%$ de los consumos las cuotas son mensuales. En este sentido, es relevante recordar que la población analizada recibe la mayor parte de sus ingresos (59\% no tiene otro trabajo) también en forma mensual (tanto el programa Argentina Trabaja como la Asignación Universal por Hijo se abonan mensualmente).

\footnotetext{
14 A inicios de 2014 se hizo sentir la falta de actualización de los montos percibidos por los cooperativistas en relación con la inflación.

${ }^{15}$ Figueiro señala que "La precariedad laboral y la informalidad es "castigada" mediante recargos, tasas de interés muy superiores a la de los bancos o, en los casos extremos, directamente la exclusión en el acceso a ciertos bienes". (2010, p.422).
} 
Tabla 2.

Modalidad temporal de los planes de cuotas de los bienes y servicios no básicos adquiridos

\begin{tabular}{lc}
\hline Rango del plan de cuotas (\# de cuotas) & Porcentaje \\
\hline Hasta 3 & $11 \%$ \\
Entre 3 y 6 & $29 \%$ \\
Entre 7 y 11 & $11 \%$ \\
12 & $37 \%$ \\
Entre 13 y 17 & $3 \%$ \\
Más de 18 & $9 \%$ \\
Total & $\mathbf{1 0 0 \%}$ \\
\hline
\end{tabular}

Nota: * De 15 productos no saben o no responden el número de cuotas.

Fuente: elaboración propia con base en la encuesta aplicada, Cisba. 2013-2014.

En cuanto a la duración de los planes de cuotas, como muestra la Tabla $\underline{2}$, encontramos que el $29 \%$ de los consumos analizados se realizaron en planes de 4 a 6 cuotas y el 37\% en planes de 12 cuotas, lo cual emparenta estos sistemas con la estructura de financiamiento más extendida en el resto de la sociedad argentina, donde los plazos estándares son los de 3, 6 y 12 cuotas mensuales. No hemos podido discriminar de qué modo y en qué medida la oferta de este tipo de planes de cuotas se vincula con los planes de financiamiento del consumo promovidos por el gobierno nacional para estimular de demanda interna.

Tabla 3.

Monto de las cuotas de los bienes y servicios no básicos adquiridos en cuotas

\begin{tabular}{lc}
\hline Rango del monto de las cuotas en pesos* & Porcentaje \\
\hline Menos de 100 & $6 \%$ \\
Entre 101 y 300 & $43 \%$ \\
Entre 301 a 500 & $35 \%$ \\
Entre 501 y 700 & $9 \%$ \\
Entre 701 y 1000 & $6 \%$ \\
Mayor de 1000 & $2 \%$ \\
Total & $\mathbf{1 0 0 \%}$ \\
\hline
\end{tabular}

Nota: *de 9 productos no saben o no responde el valor de la cuota.

Fuente: elaboración propia con base en la encuesta aplicada, Cisba, 2013-2014.

Por otro lado, cuando observamos los montos de las cuotas pagadas también se advierten ciertas regularidades. En el $78 \%$ de los consumos analizados los encuestados pagan cuotas de entre $\$ 101$ y $\$ 500$, como se aprecia en la Tabla $\underline{3}$. El 43\% pagó cuotas de entre $\$ 101$ y $\$ 300$ y el $35 \%$ de entre $\$ 301$ y $\$ 500$. Solamente el $17 \%$ pagó cuotas de más de $\$ 501$ y tan solo el $6 \%$ pagó cuotas de menos de $\$ 100$. El monto de estas cuotas, por sí mismo, da una idea del universo de bienes a los que la población que estamos estudiando tiene acceso.

58

Renato Mauricio Fumero e lara Nadina Hadad

(c) (i) 8 (O)

Revista Economía y Sociedad by Universidad Nacional is licensed under a CreativeCommons Reconocimiento-NoComercial- 
Considérese que en 2014 los ingresos percibidos por los beneficiarios del programa Argentina Trabaja; es decir, la población estudiada, eran de \$2.000; en 2015, pasaron a ser de \$2.600 y, recientemente, en 2016 representan un monto de \$3.120. Para tener una referencia más abarcativa referida al valor de las cuotas pagadas por estos bienes, puede considerarse que en enero del año 2014 el Salario Mínimo Vital y Móvil ${ }^{16}$ era de \$3.600, y en septiembre del mismo año alcanzó los \$4.400, fecha para la cual el dólar estaba comercializándose a \$8,33 para la compra y en $\$ 8,41$ para la venta.

El análisis de las fuentes de crédito para estos consumos ofrece datos importantes para tratar de caracterizar la estructura de financiamiento que se encuentra actualmente operando. Del total de los consumos, como se exhibe en la Tabla $\underline{4}$, tres de cada cuatro fueron financiados por los mismos locales de electrodomésticos y por las tarjetas de crédito. Este dato ofrece una imagen contundente respecto de la institucionalización de la financiarización en los sectores populares. Por sí mismo bastaría para contestar a los imaginaros sociales que asocian la estructura de consumos de este grupo con esquemas altamente informales, personales o, en el peor de los casos, irracionales y anómicos.

Tabla 4.

Persona o entidad dueña de la deuda contraída en la adquisición del bien o servicio no básico pagado en cuotas

\begin{tabular}{lc}
\hline \multicolumn{1}{c}{ ¿Con quién se endeudó? } & Porcentaje \\
\hline El banco & $1 \%$ \\
Financiera & $2 \%$ \\
Con tarjeta de crédito & $17 \%$ \\
Con tarjeta de crédito no bancaria & $1 \%$ \\
Negocios de electrodomésticos & $58 \%$ \\
Un familiar o amigo & $2 \%$ \\
Un prestamista & $2 \%$ \\
No identificado & $2 \%$ \\
Otro & $12 \%$ \\
NS/NR & $4 \%$ \\
Total general & $100 \%$ \\
\hline
\end{tabular}

Fuente: elaboración propia con base en la encuesta aplicada, Cisba, 2013-2014.

Ahora bien, cabe destacar que el $58 \%$ de los consumos analizados fue financiado directamente por los locales de electrodomésticos donde se compraron los productos (mientras que solo el $17 \%$ del total fue financiado por las tarjetas de crédito). Este dato habilita una agenda de investigación para pensar la forma específica en que se construye el sujeto endeudado popular

\footnotetext{
${ }^{16}$ El Salario Mínimo Vital y Móvil es el piso salarial que convienen en establecer dentro del Consejo Nacional de Empleo, la Productividad y el Salario (conocido popularmente como "Consejo del Salario"), el gobierno nacional, los sindicatos y las cámaras empresariales. Su fijación está establecida en el texto constitucional, en el artículo 14 bis.
} 
en relación con las casas de electrodomésticos, que no constituyen un espacio de consumo homogéneo. Como ha advertido Wilkis (2014), el funcionamiento financiero de las grandes cadenas de electrodomésticos y de los locales barriales del rubro ubicados se distingue drásticamente; mientras que los primeros ofrecen instrumentos formales de crédito, los segundos diseñan sus propias estrategias e instrumentos informales. Un estudio de las formas de producción subjetiva del sujeto endeudado popular en nuestro país debería poder explicar la articulación que durante los últimos años se ha dado en torno al rubro electrodomésticos, entre exigencias de distinción, estructuras de financiamiento y políticas públicas de estímulo al consumo.

Aunque en términos comparativos pueda parecer poco importante, en sí misma la presencia de las tarjetas de crédito en los consumos de los sectores populares es también un dato fundamental reciente, y cuya incidencia puede proyectarse que será cada vez más importante ${ }^{17}$. El avance de las tarjetas como instrumento financiero quizás constituya el dato más significativo en función de argumentar la hipótesis con la que iniciamos esta sección, respecto de considerar la financiarización de los sectores populares como la consecuencia de una mutación estructural en el desarrollo del capitalismo. Wilkis (2014) ha señalado que "(...) las tarjetas de crédito ya no portan su tradicional signo distintivo de clase: se han vuelto plebeyas" (p. 233). Esta afirmación ha sido corroborada por estudios realizados en otros países de la región ${ }^{18}$.

Los datos procesados sobre el monto de la tasa de interés que paga la población analizada ofrecen los resultados más preocupantes de nuestro estudio. Del análisis de 81 consumos registrados surge que los encuestados han debido afrontar créditos con tasas de interés promedio del 89\%; más allá incluso del 57\% que había observado Figueiro (2010), en un estudio sobre el financiamiento ofrecido por casas de electrodomésticos e instituciones que ofrecen préstamos inmediatos. Se trata de una diferencia sustancial con la que afrontan otros sectores sociales que pueden financiar sus consumos al $0 \%$ de interés anual o con tasas que no van más allá del 20\%. Una situación especialmente gravosa en función de las formas de financiamiento a las que acceden los sectores populares, ya que "...tienen incorporado la obligación de pago de deudas, sin que puedan negociar o tener derechos protegidos" (Roig, 2017, p. 90).

\footnotetext{
${ }^{17}$ Barros (2009) ha ofrecido datos certeros sobre la expansión del acceso a tarjetas de crédito en Chile. Para el caso Argentino aún falta un estudio similar.

${ }^{18}$ Véase para el caso de Chile, Montero y Tarziján (2010), Barros (2009) y Ossandón et al. (2012). Para el caso brasilero puede consultarse Kumar (2004).

60
}

Renato Mauricio Fumero e lara Nadina Hadad 
Tabla 5.

Uso de tarjeta de crédito en los consumos

\begin{tabular}{lc}
\multicolumn{1}{c}{ Usa tarjeta de crédito } & Porcentaje \\
\hline No & $68 \%$ \\
Sí & $25 \%$ \\
NS/NR & $7 \%$ \\
Total & $100 \%$ \\
\hline
\end{tabular}

Fuente: elaboración propia con base en la encuesta aplicada, Cisba, 2013-2014.

Tabla 6.

Titularidad de la tarjeta de crédito utilizada en los consumos

\begin{tabular}{lc}
\hline \multicolumn{1}{c}{ De quién es la tarjeta } & Porcentaje \\
\hline Propia & $27 \%$ \\
Familiar & $53 \%$ \\
Tarjeta extensión de un tercero & $13 \%$ \\
NS/NR & $7 \%$ \\
Total & $\mathbf{1 0 0 \%}$ \\
\hline
\end{tabular}

Fuente: elaboración propia con base en la encuesta aplicada, Cisba, 2013-2014.

Como se aprecia en la Tabla $\underline{5}$, de las 183 personas consultadas solo el $25 \%$ ha afirmado utilizar tarjeta de crédito. De este porcentaje, como muestra la Tabla $\underline{6}$, más de la mitad (el 53\%) ha manifestado que la tarjeta pertenece a un familiar. Estos préstamos hablan de cierta mutualización del dinero dentro del hogar (Weber, 2005) que, como fue evidenciado en el caso chileno (Ossandón et al., 2012), articulan "circuitos comerciales" (Zelizer, 2005). Apenas el 27 \% de los consultados confirmó ser titular de la misma y el restante $13 \%$ corresponde a quienes poseen extensión de una tarjeta perteneciente a un tercero. De igual modo, los créditos personales no siempre se obtienen a "nombre propio", sino que es usual gestionarlo a nombre de algún pariente. En efecto, del total de los consumos en cuotas que hemos relevado (178 sobre una base de $282^{19}$ ), constatamos que solo el $50 \%$ es titular formalmente del instrumento financiero. Es decir, a través del uso de la propia tarjeta de crédito (siendo titular) o de un crédito personal solicitado y ofrecido por el comercio.

Si contemplamos esta práctica de préstamos de tarjetas de crédito junto al dato exhibido al inicio de esta sección referido a los préstamos de dinero entre familiares y amigos (el $31 \%$ de los entrevistados declaró prestar dinero y el 54 \% de estos dijo prestarlo a familiares), es posible comenzar a reconstruir la escala doméstica de las tramas de financiamiento de la economía

\footnotetext{
${ }^{19}$ Tomando como base los 282 consumos analizados, es posible afirmar que el 63 por ciento ha sido realizado a crédito, mientras el 33.3 por ciento, al contado. Los electrodomésticos, ropa, celulares y muebles son los bienes mayormente adquiridos en cuotas, pero también -aunque en mucha menor medida-, al contado.
} 
popular, la cual requiere aun de un estudio atento a sus dinámicas y formas de jerarquización específica. Wilkis (2014) remarca que en la socialización de los instrumentos también se produce la "socialización de las deudas" (p. 246) entre los miembros del hogar, con el fin de sostener la reproducción material. Haciendo hincapié en la dinámica familiar, advierte que es la familia en su conjunto la que se endeuda o, en otras palabras, la que gestiona las deudas existentes en el presupuesto doméstico. Pero esta consideración pareciera no atender a los modos en que al interior de las familias se construyen roles subjetivos jerárquica $y$ funcionalmente diferentes. Es decir, quien puede obtener un crédito o prestar una tarjeta de crédito ocupa una posición en la economía popular diferente de quien requiere de la asistencia de aquel para poder acceder a los consumos. Las estrategias financieras familiares se constituyen como corolario de las formas de acceso al crédito. A este respecto, el acceso de las mujeres al crédito (a través de una tarjeta o crédito personal) es un hecho que no puede separarse del entrecruzamiento de dos fenómenos: la extensión del mercado del crédito y la política social (Wilkis, 2014). Del total de personas encuestadas, el 66\% correspode a mujeres, de las cuales el $52 \%$ recibe la AUH. Esta transferencia monetaria condicionada, a diferencia del ingreso social por trabajo recibido en el marco del programa Argentina Trabaja, ha sido señalada como sustituto válido de documentos de identificación económica (Laferté, 2014). Efectivamente, la Asignación Universal por Hijo (AUH) es ampliamente utilizada como "garantía económica" para acceder a un crédito o a una tarjeta.

\section{Conclusión}

Este artículo ha intentado realizar un aporte a la comprensión de la fisonomía e incidencia de los consumos financiarizados de los sectores populares. En este sentido, los datos analizados han buscado confirmar la apuesta formulada al inicio de este trabajo: los consumos financiarizados ofrecen una perspectiva analítica renovada para pensar la economía popular. Decíamos que confiábamos en que el modo de producir esta aproximación consistía en proponer una doble inscripción, estructural y subjetiva, de las prácticas e instituciones sociales analizadas. Ahora bien, en líneas generales, los resultados alcanzados ofrecen una caracterización precisa de los consumos financiarizados de los sectores populares:

- Son consumos orientados mayormente a la adquisición de productos electrónicos y, en menor medida, de ropa; dos áreas que el Estado nacional estimuló particularmente durante los años estudiados, a través de planes de financiamiento que alcanzaban las prácticas formalizadas de consumos

- Son consumos que, a través de los sistemas de financiamiento utilizados, muestran la penetración que tienen en la economía popular los negocios de electrodomésticos y las tarjetas de crédito

- Son consumos que revelan la existencia de una vida financiera de la economía popular cuya singularidad surge del modo en que se traman en ella prácticas e

62

Renato Mauricio Fumero e lara Nadina Hadad

$$
\text { (†) } 90
$$

Revista Economía y Sociedad by Universidad Nacional is licensed under a CreativeCommons Reconocimiento-NoComercial- 
instituciones generales (tarjetas de crédito, créditos al consumo, etc.) con prácticas e instituciones específicas (préstamos de dinero y tarjetas de crédito entre familiares).

- Son consumos que, de este modo, nos colocan en situación de explorar el funcionamiento de las economías domésticas de los sectores populares y, específicamente, el modo en que los ingresos y los consumos afectan la configuración de las familias.

- Son consumos, finalmente, que dan cuenta de la existencia de un fenómeno social extendido y teóricamente desafiante. Al mismo tiempo, estos consumos alertan sobre la necesidad de políticas públicas orientadas que impidan que sus participantes se vean afectados por situaciones abusivas (por ejemplo, en la fijación de los precios o condiciones de financiamiento).

En términos estructurales, el trabajo aporta datos precisos y circunstanciados para profundizar el análisis de la inscripción específica de los sectores populares en las tramas financieras y de consumo. El tipo de bienes y las modalidades de financiamiento y, especialmente, la magnitud de las tasas de interés, recortan, a través de los consumos, a un sujeto social particular. Complementan aquellos datos el estudio de las prácticas que animan una vida financiera que reescribe las condiciones formales impuestas por las tarjetas de crédito y los negocios que ofrecen compras en cuotas. Hemos tratado de orientar la indagación sobre este aspecto, en dirección a la pregunta por el tipo de subjetividad que producen estas prácticas. Explícitamente, el análisis realizado se ofrece como un ejercicio reflexivo que deja abiertas interrogantes que intentaremos responder en el futuro.

\section{Referencias}

Balzarotti, V. y Anastasi, A. (2013). ¿La competencia por deudores recién incorporados perjudica el acceso al crédito? Análisis en un contexto de alto riesgo y baja bancarización. Ensayos Económicos, (69), 101-149. Recuperado de http://www.bcra.gob.ar/Institucional/Resumen.asp?id=250\&prevPage=1

Barros, P. (2009) ¿Tres cuotas, precio contado? Observaciones sobre el endeudamiento de los chilenos". En: Fuentes, C. (Ed.). Chile 2008: Percepciones y actitudes sociales. 4으 Informe de Encuesta Nacional UDP. Recuperado de http://www.icso.cl/images/documentos/encuestaudp/2008/informe.pdf

Bataille, G. (2008). La conjuración sagrada. Ensayos 1929-1939. Buenos Aires, Argentina: Adriana Hidalgo Editora. Recuperado de https://andre69999.files.wordpress.com/2012/03/bataille-georges-la-conjuracionsagrada-ensayos-1929-19391.pdf 
Bercovich, A. (2016). Endeudar al soberano. Revista Crisis, (27), 10-12. Recuperado de http://www.revistacrisis.com.ar/notas/endeudar-al-soberano

Chena, P. (2017). La economía popular y sus relaciones fundantes. En E. Pérsico et. al, Economía Popular: Los desafíos del trabajo sin patrón (pp. 41-62). Buenos Aires, Argentina: Colihue. Recuperado de https://www.boutiquedellibro.com.ar/9789876843119/Econom\%C3\%ADa+Popular+ +Los+Desaf\%C3\%ADos+Del+Trabajo+Sin+Patr\%C3\%B3n/

Cielo, C. y Antequera, N. (2012). Ciudad sin Fronteras: La multilocalidad rural urbana en Bolivia. Eutopía, (3), 11-29. Recuperado de file:///E:/Descargas/DialnetciudadSinFronteraLaMultilocalidadUrbanoruralEnBoli-5040170.pdf

De Certeau, M. (1996). Invención de lo cotidiano I. Artes de hacer. Recuperado de https://books.google.co.cr/books/about/La_Invenci\%C3\%B3n_de_lo_cotidiano.html ?hl=es\&id=iKqK5OfkLnUC\&redir_esc=y

Deleuze, G. (1995). Conversaciones 1972-1990. Valencia, España: Pre-Textos (trad. José Luis Pardo). Recuperado de http://bibliotecaparalapersonaepimeleia.com/greenstone/collect/libros1/index/assoc/HASHa26a.dir/doc.pdf

D’Onofrio, F. (2008) Crédito al consumo tras la crisis: el boom del consumer finance en Argentina (tesis de Licenciatura). Argentina: Universidad Nacional de Mar del Plata. Recuperado de http://nulan.mdp.edu.ar/634/1/donofrio f.pdf

Epstein, G. (Ed.) (2005). Financialization and the World Economy. Recuperado de https://books.google.co.cr/books/about/Financialization and the World Economy. html?id=fZnQf2IBO4YC\&redir esc=y

Figueiro, P. (2010). Disponer las prácticas. Consumo, crédito y ahorro en un asentamiento del Gran Buenos Aires. Civitas - Revista de Ciências Sociais, 10 (3), 410-429. Recuperado de http://revistaseletronicas.pucrs.br/ojs/index.php/civitas/article/view/8339

Figueiro, P. (2013). Lógicas sociales del consumo. El gasto improductivo en un asentamiento bonaerense. https://books.google.co.cr/books/about/L\%C3\%B3gicas sociales del consumo.htm l?id=Ph23oQEACAAJ\&redir esc=y

Figueiro, P. (2016). El sujeto Actual. Revista Márgenes, (3). Recuperado de http://www.unsam.edu.ar/margenes/?p=940

64

Renato Mauricio Fumero e lara Nadina Hadad

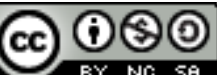

Revista Economía y Sociedad by Universidad Nacional is licensed under a CreativeCommons Reconocimiento-NoComercial- 
Gago, V. (2012). La Salada, ¿un caso de globalización desde abajo? Revista Nueva Sociedad, (241). Recuperado de http://nuso.org/articulo/la-salada-un-caso-de-globalizaciondesde-abajo-territorio-de-una-nueva-economia-politica-transnacional/

Gago, V. (13 de octubre del 2013). El consumo como marca de época. Le Monde Diplomatique. Recuperado de http://anarquiacoronada.blogspot.com/2013/11/el-consumopopular-como-marca-de-epoca.html

Giraldo, C. (2013). Política Social Contemporánea en América Latina. Entre el asistencialismo y el mercado. Recuperado de https://books.google.co.cr/books/about/Pol\%C3\%ADtica_social_contempor\%C3\%A 1nea en Am\%C3\%A9r.html?id=HVQTnQEACAAJ\&redir esc=y

Giraldo, C. (2017) Los sectores populares en Colombia: Excluidos del contrato social. En E. Pérsico et. al, Economía Popular: Los desafíos del trabajo sin patrón (pp. 191-199). Buenos Aires, Argentina: Colihue. Recuperado de https://www.boutiquedellibro.com.ar/9789876843119/Econom\%C3\%ADa+Popular+ +Los+Desaf\%C3\%ADos+Del+Trabajo+Sin+Patr\%C3\%B3n/

Hornes, M. (2014). Transferencias condicionadas y sentidos plurales: el dinero estatal en la economía de los hogares argentinos. Revista Antípoda, (18), 61-83. Doi: https://doi.org/10.7440/antipoda18.2014.04

Kessler, G. (2014). Controversias sobre la desigualdad. Argentina, 2003-2013. Buenos Aires, Argentina: Fondo de Cultura Económica. Recuperado de https://es.scribd.com/doc/257828162/Controversias-sobre-la-desigualdad-pdf

Kumar, A. (Coord.). (2004), Brasil: acesso a serviços financeiros. Recuperado de http://www.ipea.gov.br/portal/index.php?option=com content\&view=article\&id=5 $\underline{487}$

Laferté, G. (2014). Economic Identification: A contribution to a Comparative Socio-History of Credit Market. Economic Sociology, 15(3), 5-11. Recuperado de https://www.econstor.eu/bitstream/10419/156034/1/vol15-no03-a2.pdf

Lazzarato, M. (2013). La fábrica del hombre endeudado. Ensayo sobre la condición neoliberal. Buenos Aires: Amorrortu. Recuperado de https://es.scribd.com/doc/212583321/Lazzarato-Maurizio-La-Fabrica-Del-HombreEndeudado-Completo-Smallpdf-com 
Montero, J.P. \& Tarziján, J. (2010). El Ëxito de las Casas Comerciales en Chile: ¿Regulación o Buena Gestión? (Documento de Trabajo, 565). Recuperado del sitio web del Banco Central de Chile: http://si2.bcentral.cl/public/pdf/documentostrabajo/pdf/dtbc565.pdf

Müller, L. (2009). Então, eu fui à luta! Repensando as representações e práticas económicas de grupos populares a partir de uma trajetória de ascensão social. Política/Sociedade, 8(15), 147-172. Doi: https://doi.org/10.5007/2175-7984.2009v8n15p145

Müller, L. (2014). Negotiating debts and gifts: financialization policies and the economic experiences of low-income social groups in Brazil. Vibrant: Virtual Brazilian Anthropology, 11(1), 191-221. Doi: https://doi.org/10.1590/S1809$\underline{43412014000100007}$

Ossandón, J. (Ed.) (2011). Destapando la Caja Negra. Sociologías de los créditos de consumo en Chile. Recuperado de http://www.icso.cl/wpcontent/uploads/2012/01/Destapando-la-Caja-Negra-Sociolog\%C3\%ADa-de-losCr\%C3\%A9ditos-de-Consumo-Editado-por-Jos\%C3\%A9-Ossand\%C3\%B3n-Enero2012.pdf

Ossandón, J., Ariztía, T., Barros, M. \& Peralta, C. (2012). The Economy of the Quota: The Financial Ecologies and Commercial Circuits of Retail Credit Cards in Santiago, Chile. Recuperado de http://blog.imtfi.uci.edu/2012/11/the-economy-of-quotafinancial.html

Polanyi, K. (1976). El sistema económico como proceso institucionalizado. En M. Godelier (Comp.), Antropología y economía (pp. 155-178). Recuperado de https://www.casadellibro.com/libro-antropologia-yeconomia/9788433906069/289628

Roig, A. (2017). Financiarización y derechos de los trabajadores de la economía popular. En E. Pérsico et. al, Economía Popular: Los desafíos del trabajo sin patrón (pp. 87-102). Buenos Aires, Argentina: Colihue. Recuperado de https://www.boutiquedellibro.com.ar/9789876843119/Econom\%C3\%ADa+Popular+ +Los+Desaf\%C3\%ADos+Del+Trabajo+Sin+Patr\%C3\%B3n/

Roig, A. (2015). Separar de sí, separar para sí: las prácticas de ahorro domésticas en sectores populares urbanos argentinos. En A. Wilkis y A. Roig (Eds.), El laberinto de la moneda $y$ las finanzas (pp.195-210). Buenos Aires, Argentina: Biblos. Recuperado de https://www.waldhuter.com.ar/libreria/Papel/9789876913966/EL+LABERINTO+DE+ LA+MONEDA+Y+LAS+FINANZAS

66

Renato Mauricio Fumero e lara Nadina Hadad

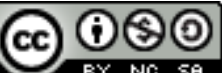

Revista Economía y Sociedad by Universidad Nacional is licensed under a CreativeCommons Reconocimiento-NoComercial- 
Van Der Zwan, N. (2014). Making sense of financialization. Socio-economic Review, 12 (1), 99 129. Doi: https://doi.org/10.1093/ser/mwt020

Villarreal, M. (Comp.) (2004). Antropología de la deuda. Crédito, ahorro, fiado, ahorro y prestado en las finanzas cotidianas. Recuperado de https://www.amazon.com.mx/antropologia-credito-prestado-finanzascotidianas/dp/9707014822

Weber, F. (2005). Le sang, le nom, le quotidien. Une sociologie de la parenté pratique. París, Francia: Aux Lieux D'être. Recuperado de https:///homme.revues.org/3030

Wilkis, A. (2013). Las sospechas del dinero. Moral y economía en el mundo popular. Recuperado de http://www.planetadelibros.com.ar/las-sospechas-del-dinero-libro-132258.html

Wilkis, A. (2014). Sociología del crédito y economía de las clases populares. Revista Mexicana de $\begin{array}{lllll}\text { Sociología, } & 76 & (2), & 225-252 . & \text { Recuperado de }\end{array}$ http://www.redalyc.org/articulo.oa?id=32130485004

Wilkis, A. y Roig, A. (Eds.). (2015). El laberinto de la moneda y las finanzas. Buenos Aires, Argentina: Biblos. Recuperado de http://www.marcialpons.es/libros/el-laberinto-dela-moneda-y-las-finanzas/9789876913966/

Zelizer, V. (2005). Circuits within capitalism. En V. Nee; R. Swedberg (Eds.), The economic sociology of capitalism. Recuperado de http://press.princeton.edu/titles/7995.html 